\title{
Asymptotic Analysis of the Paradox in Log-Stretch Dip Moveout
}

\author{
Xin-She Yang \\ Department of Applied Mathematics, University of Leeds, Leeds LS2 9JT, UK. \\ Binzhong Zhou \\ CSIRO Division of Exploration and Mining, P.O.Box 883, Kenmore, QLD 4069, AUSTRALIA.
}

\begin{abstract}
There exists a paradox in dip moveout (DMO) in seismic data processing. The paradox is why Notfors and Godfrey's approximate time log-stretched DMO can produce better impulse responses than the full $\log$ DMO, and why Hale's f-k DMO is correct although it was based on two inaccurate assumptions for the midpoint repositioning and the DMO time relationship? Based on the asymptotic analysis of the DMO algorithms, we find that any form of correctly formulated DMO must handle both space and time coordinates properly in order to deal with all dips accurately. The surprising improvement of Notfors and Godfrey's log DMO on Bale and Jakubowicz's full log DMO was due to the equivalent midpoint repositioning by transforming the timerelated phase shift to the space-related phase shift. The explanation of why Hale's f-k DMO is correct although it was based on two inaccurate assumptions is that the two approximations exactly cancel each other in the f-k domain to give the correct final result.
\end{abstract}

Citation detail: X. S. Yang and B. Zhou, Asymptotic analysis of the paradox in log-stretch dip moveout, Geophys. Res. Lett., 27(3), 441-444 (2000).

\section{INTRODUCTION}

Dip Moveout (DMO) technique has been widely used in seismic data processing over the past decade and many different algorithms have been developed [Hale, 1984; Bale and Jakubowicz, 1987; Notfors and Godfrey, 1987; Liner, 1990; Gardner, 1991; Black et al., 1993; Zhou et al., 1996]. Hale's DMO is accurate for all reflector dips and has become an industrial standard, but it is computational expensive and temporally non-stationary. In all the methods, a logarithmic time-stretching technique [Bolondi et al., 1982] is widely used, probably due to its computational efficiency and easy implementation. The impulse response produced by Bale and Jakubowicz's full log-stretch operator (hereafter referred to as Bale's full $\log$ DMO) in the frequency-wavenumber (fk) domain is inaccurate although no approximations were made in the mathematical formulations. The impulse response is surprisingly improved by Notfors and Godfrey's approximate log-stretch scheme (hereafter referred to as Notfors' log DMO). Bale's inaccurate DMO impulse responses reveal that Hale's DMO derivation had some inappropriate assumptions. To improve the DMO impulse responses, an exact logstretch DMO was derived by transforming Hale's time log-stretch impulse response into the Fourier domain [Liner, 1990] . Black et al. [1993] provided another derivation of the more reliable f-k DMO by treating the reflection-point smear from dipping reflectors correctly. Based on Black's DMO relationship, Zhou et al. $[1995,1996]$ presented a log-stretch $\mathrm{f}-\mathrm{k}$ DMO which is similar to Gardner's DMO [Gardner, 1991] but from a derivation with more direct physical insight into the DMO process. All these DMO schemes were developed to try to generate better impulse responses and to improve the computational speed, but have different corrections of Hale's subtle flaw and improving Bale's inaccurate impulse responses.

Despite extensive studies and the routine use of the DMO algorithms, there still exists a paradox which has not yet been explained satisfactorily. The paradox is why Notfors' approximate log DMO can produce better impulse responses than Bale's full log DMO, and why Hale's f-k DMO is correct although it was based on two inaccurate assumptions. Liner pointed out [Liner, 1990] that Bale and Jakubowicz's DMO derivation implicitly assumes that the Fourier transform frequency in the log-stretch domain is time-independent. Black et al. attributed Hale's subtle flaw to the lack of midpoint change and the 
consequently improper treatment of the reflectionpoint smear from dipping reflectors. Zhou et al. suggested that the approximations from the nonrepositioned midpoint and from the incorrect time relationship before and after DMO may counteract each other in the f-k domain to make the final result correct, but they may not cancel each other in the logstretch f-k domain. In this paper, we analyse these viewpoints and compare DMO algorithms to find out what is really responsible for the inaccurate impulse responses of Bale's full log DMO scheme. For convenience in the following discussion, only commonoffset DMO forms will be analysed although similar methodology can be also applied to common-shot DMO analysis.

\section{ASYMPOTIC ANALYSIS OF PHASE SHIFT FUNCTIONS}

We assume a constant-velocity medium and follow the notations used earlier [Hale, 1984; Notfors and Godfrey, 198\%; Black et al., 1993; Zhou et al., 1996]. The heuristic DMO mapping can be expressed by

$$
P_{0}\left(t_{0}, x_{0}, h\right)=P_{n}\left(t_{n}, x_{n}, h\right),
$$

where $P_{n}\left(t_{n}, x_{n}, h\right)$ is a normal-moveout (NMO) corrected input section and $P_{0}\left(t_{0}, x_{0}, h\right)$ is a zero-offset DMO output section. $h$ is the half offset, $t_{n}$ and $x_{n}$ are time and space in the NMO-corrected domain respectively, and $t_{0}$ and $x_{0}$ are the time and space in DMO-corrected domain, respectively. The time logstretch transform pair [Bolondi et al., 1982; Bale \&6 Jakubowicz 1987]

$$
\tau=\ln \left(t / t_{c}\right) \text { and } t=t_{c} e^{\tau}
$$

transforms the time coordinates from $\left(t_{0}, t_{n}\right)$ to the log-stretch variables $\left(\tau_{0}, \tau_{n}\right) . t_{c}$ is the minimum cutoff time introduced to avoid the singularity of the logarithm at zero.

The DMO processing algorithm can be divided into the following five steps [Liner, 1990; Gardner, 1991; Hale, 1991]: 1) transform NMO corrected input data $P_{n}\left(t_{n}, x_{n}, h\right)$ to $P_{n}\left(\tau_{n}, x_{n}, h\right)$ by the time log stretch relation (2), 2) 2-D FFT of $P_{n}\left(\tau_{n}, x_{n}, h\right)$ over $\tau_{n}$ and $x_{n}$ to $\left.P_{n}(\Omega, k, h), 3\right)$ use the phase factor for filtering in the $(\Omega, k)$ domain to get $\left.P_{0}(\Omega, k, h), 4\right)$ inverse $2-\mathrm{D}$ FFT of $P_{0}(\Omega, k, h)$ over $\Omega$ and $k$ to $P_{0}\left(\tau_{0}, x_{0}, h\right)$, and $5)$ inverse $\log$ stretch of $P_{0}\left(\tau_{0}, x_{0}, h\right)$ by the inverse relation of equation (2) to get the output $P_{0}\left(t_{0}, x_{0}, h\right)$. Of all the available log stretch DMO algorithms, the same transform relation (2) is used in steps 1) and 5), and the two-dimensional FFT transform pair is also used in steps 2) and 4). The main difference between algorithms is thus the multiplying phase factor in step 3 ). The following discussion will therefore focus on the analysis of the geometry-determining phase factor in different log-stretch DMO algorithms. As we will see, all the phase shift functions depend mainly on the variable

$$
\xi=\frac{h k}{\Omega} .
$$

Mathematically speaking, $\xi$ can vary from 0 (zero dip at the centre of the DMO ellipse) to $\infty$ (very shallow, steeply dipping).

\section{Bale's Full Log DMO}

From Hale's DMO formulation, Bale and Jakubowicz [1987] derived the full log stretch DMO

$$
P_{0}(\Omega, k, h)=e^{-i \frac{1}{2} \Omega \ln \left(1-\xi^{2}\right)} P_{n}(\Omega, k, h),
$$

However, this algorithm does not produce good impulse responses. Liner [1990] attributed this feature to the fact that Bale and Jakubowicz's derivation implicitly assumes that $\Omega$ is not time-dependent. In fact, Bale's phase shift function

$$
\Phi_{F}=-\frac{1}{2} \Omega \ln \left(1-\xi^{2}\right)
$$

is only physically meaningful when $\xi<1$ (near zero dips). It becomes complex if $\xi \geq 1$ (shallow, steep dips) which is responsible for the inverted Gaussian shape.

\section{Notfors' Log DMO}

An approximation to (4) was derived by Notfors and Godfrey [1987]

$$
P_{0}(\Omega, k, h)=e^{i \Omega\left(\sqrt{1+\xi^{2}}-1\right)} P_{n}(\Omega, k, h),
$$

which is based on two approximations: (1) the independence of $\Omega$ and $\tau_{n}$, and $(2)(h k / \Omega)^{2}<<1$. With these approximations, Bale's phase shift function becomes Notfors' phase shift function

$$
\Phi_{N}=\Omega\left(\sqrt{1+\xi^{2}}-1\right),
$$

which is meaningful for any value of $\xi$ (all reflector dips). This derivation implicitly extends the definition range of $\xi$ although the approximations are based on $\xi<<1$. It is this second approximation that makes Notfors' log DMO produce better impulse responses than Bale's full log DMO (4). 


\section{Liner's Log DMO}

The exact log stretch DMO derived by Liner [1990] is

$$
P_{0}(\Omega, k, h)=\frac{e^{i\left[\Omega \Delta_{s}-k y_{s}\right]}}{\left(1+\beta_{s}^{2}\right)^{1 / 2}} P_{n}(\Omega, k, h)
$$

where

$$
\begin{gathered}
\beta_{s}=y_{s} / h, \\
\Delta_{s}=\frac{1}{2} \ln \left(1-\beta_{s}\right),
\end{gathered}
$$

and the stationary point is

$$
y_{s}=\frac{h}{2 \xi}\left(1-\sqrt{1+4 \xi^{2}}\right) .
$$

Liner's derivation introduces $\log$ stretch variables into Hale's $(t, x)$ elliptical response rather than into the NMO equation, as was done earlier by Bale and Jakubowicz [1987]. The formula (8) does yield the correct impulse response geometry [Liner, 1990; Zhou et al., 1996].

\section{Zhou's Log DMO}

Zhou et al. [1996] presented an accurate log stretch $\mathrm{DMO}$

$P_{0}(\Omega, k, h)=e^{i \frac{1}{2} \Omega\left[\sqrt{1+4 \xi^{2}}-1-\ln \left[\frac{1}{2}\left(\sqrt{1+4 \xi^{2}}+1\right)\right]\right.} P_{n}(\Omega, k, h)$

which is equivalent to Gardner's results except for a sign difference on the phase term due to a different definition of the 2-D Fourier transform in Gardner's formulation. Zhou et al. [1996] derived their result based on the DMO relationships given by Black et al. [1993]. It is easy to check that the phase shift in equation (12) is identical to that in equation (8). Therefore, Zhou's log DMO and Liner's log DMO will produce the same impulse response geometry. The only difference is that Zhou's DMO will yield slightly larger amplitudes than Liner's DMO because (8) has an amplitude factor $1 /\left(1+\beta_{s}^{2}\right)^{1 / 2} \leq 1$. For convenience in the following analysis, we will refer to Liner's log DMO and Zhou's log DMO as the exact $\log \mathrm{DMO}$, and will use the simpler phase shift expression

$$
\Phi_{E}=\frac{1}{2} \Omega\left[\sqrt{1+4 \xi^{2}}-1-\ln \left[\frac{1}{2}\left(\sqrt{1+4 \xi^{2}}+1\right)\right],\right.
$$

which is accurate for all reflector dips.

All the phase shift functions (5), (7) and (13) are monotonically increasing, therefore, we discuss only two asymptotic cases.
Case 1: $\xi<<1$

For $\xi \rightarrow 0$, equations (5), (77), (13) all become

$$
\Phi_{F, N, E} \approx \frac{1}{2} \Omega \xi^{2},
$$

which means that all these log-stretch DMO algorithms are asymptotically equivalent near zero dips $(\xi \rightarrow 0)$ and will yield virtually the same impulse response geometry near the center of the DMO ellipse.

Case 2: $\xi>>1$

As the reflector dips become shallow and steep (for $\xi>>1$ ), expression (5) will be invalid, but (7) and (13) can be asymptotically approximated as

$$
\Phi_{N} \approx \Omega \xi,
$$

and

$$
\Phi_{E} \approx \Omega \xi\left(1-\frac{\ln \xi}{2 \xi}\right),
$$

respectively. Using $\frac{\ln \xi}{\xi} \rightarrow 0$ as $\xi \rightarrow \infty$, we can write (15) and (16) as a single asymptotic form

$$
\Phi_{N, E} \sim \Omega \xi
$$

which means that Notfors' log-stretch DMO and the exact log-stretch are asymptotically equivalent but they are not approximately equal due to the term $O\left(\frac{\ln \xi}{\xi}\right)$ in (16). This implies that the exact $\log$ DMO is able to deal with all reflector dips correctly, but Notfors' log DMO mishandles the shallow, steep dips although it is an improvement on Bale's DMO scheme.

\section{THE EXPLANATION OF THE PARADOX}

Based on the above analysis, we can explain the paradox of why Notfors approximate log DMO improved Bale's full log DMO, and find out the real flaw in Hale's formulation. The f-k integral for commonoffset in Hale's DMO [1984] is

$$
\begin{aligned}
& P_{0}(\omega, k, h)=\iint P_{0}\left(t_{0}, x_{0}, h\right) e^{i\left(\omega t_{0}-k x_{0}\right)} d x_{n} d t_{n} \\
& =\iint \frac{1}{A} e^{i\left(\omega t_{n} A-k x_{n}\right)} P_{n}\left(t_{n}, x_{n}, h\right) d x_{n} d t_{n},
\end{aligned}
$$

where

$$
A=\sqrt{1+\left(\frac{h}{t_{n}} \frac{d t_{0}}{d x_{0}}\right)^{2}}=\sqrt{1+\left(\frac{h k}{t_{n} \omega}\right)^{2}} .
$$


and the DMO mapping (11) and the following relations of time and space coordinate are used

$$
t_{0}=A t_{n} \quad \text { and } \quad x_{0}=x_{n},
$$

which are not strictly correct and make the DMO operator amplitude-unpreserved. The similar time and space relationships were used by Bale and Jakubowicz [1987] in their full log DMO derivation. Black et al. [1993] derived a new amplitude preserving DMO integral

$$
\begin{aligned}
& P_{0}(\omega, k, h)=\iint P_{0}\left(t_{0}, x_{0}, h\right) e^{i\left(\omega t_{0}-k x_{0}\right)} d x_{n} d t_{n} \\
& =\iint \frac{2 A^{2}-1}{A^{3}} e^{i\left(\omega t_{n} A-k x_{n}\right)} P_{n}\left(t_{n}, x_{n}, h\right) d x_{n} d t_{n} .
\end{aligned}
$$

by substituting (11) and basing on Black's correct relationship of time and space coordinates before and after DMO processing

$$
t_{0}=\frac{t_{n}}{A} \quad \text { and } \quad x_{0}=x_{n}-\frac{1}{A} \frac{h^{2}}{t_{n}} \frac{k}{\omega},
$$

where the definition of $A$ is the same as in (19). From equation (22), we can easily derive the DMO impulse ellipse (Zhou et al., 1995) while the relationships in equation (20) give an impulse point. As $A \geq 1$, Black's correct relationship (22) implies the inequality

$$
t_{0} \leq t_{n} .
$$

It is clearly seen that phase shifts in Black's DMO (21) and in Hale's DMO (18) are identical [Black et al., 1993] due to the identical phase factor $\exp \left[i\left(\omega t_{n} A-k x_{n}\right)\right]$. This implies that the two approximations resulting from the inaccurate relationships (20) exactly cancel each other in the f-k domain, and thus make the final result correct. However, the counteraction of the two approximations does not give exact cancellation in Bale's log-stretch DMO algorithm because only the time coordinate is stretched and the space coordinate remains unchanged. Therefore, we expect that the phase shift in Bale's log DMO is essentially due to the log-stretch traveltime relationship

$$
\tau_{0}-\tau_{n}=-\frac{1}{2} \ln \left(1-\xi^{2}\right) .
$$

This implies the incorrect inequalities

$$
\tau_{0} \geq \tau_{n} \quad \text { and } \quad t_{0} \geq t_{n},
$$

which contradict the correct relation (23). Notfors and Godfrey [1987] used the approximate version of (24)

$$
\tau_{0}-\tau_{n}=\sqrt{1+\xi^{2}}-1,
$$

which yields the same incorrect inequality as in (25).

From the general forms of log-stretch DMO algorithms, we know that the phase shift function

$$
\Phi=k\left(x_{n}-x_{0}\right)-\Omega\left(\tau_{n}-\tau_{0}\right),
$$

consists of two parts: a space-related phase shift $k\left(x_{n}-x_{0}\right)$ and a time-related phase shift $\Omega\left(\tau_{n}-\tau_{0}\right)$. The asymptotic analysis in the previous section reveals that the space-related phase shift is always greater than the time-related phase shift. Comparing the phase shift functions (7) and (13), we see that Notfors approximate log DMO virtually transformed the time-related phase shift into its space-related counterpart, and thus equivalently repositioned the midpoint

$$
x_{0}=x_{n}+\frac{h}{\xi}\left(1-\sqrt{1+\xi^{2}}\right),
$$

where the second term on the right hand side is similar to the expression (11) of the stationary point $y_{s}$ in Liner's exact log DMO. It is this equivalent midpoint repositioning that makes Notfors' approximate log DMO overcome Hale's subtle flaw and subsequently produces a better match to the geometry of the impulse response DMO ellipse, but the Notfors' wider geometry of the DMO ellipse shows that the shallow steep dips are still improperly treated.

\section{CONCLUSIONS}

The analysis of different log-stretch DMO algorithms has shown that any form of correctly formulated DMO must handle both space and time coordinates properly in order to deal with all dips accurately. The log-stretch DMO algorithms presented by Liner [1990] and Zhou et al. [1996] are able to treat all reflector dips correctly. Bale and Jakubowicz's full $\log$ DMO is only approximately correct for the near zero dips, and it becomes singular for shallow, steep dips. Notfors and Godfrey's approximate log DMO is a great improvement in handling all dips, but its impulse response still departs from the exact DMO ellipse, especially for shallow steep dips. The surprising improvement of Notfors and Godfrey's log DMO on Bale and Jakubowicz's full log DMO was mainly due to the equivalent midpoint repositioning by equivalently transforming the time-related phase shift to the space-related phase shift. The explanation of why Hale's f-k DMO is correct although it was based on two inaccurate assumptions is that the two approximations exactly cancel each other in the f-k domain to give the correct final result. 
Acknowledgements. The authors wish to thank the anonymous referees for their very helpful comments. This research was supported by CSIRO Exploration and Mining in Australia and the Cooperative Research Centre for Mining Technology and Equipment.

\section{REFERENCES}

Bale, R., and Jakubowicz, H., Post-stack prestack migration, 57th Ann. Mtg., Soc. Exp. Geophys., Expanded Abstract, 714-717, 1987.

Black, J. L., Schleicher, K. L., and Zhang, L., True-amplitude imaging and dip moveout: Geophysics, 58, 47-66, 1993.

Bolondi, G., Loinger, E., and Rocca, F., Offset continuation of seismic sections, Geophys. Prosp., 30, 813-828, 1982.

Gardner, G. H. F., Interpolation, crossline migration and inline depth migration of 3-D marine surveys, The SAL Annual Progress Review, 24, 57-69, 1991.

Hale, D., Dip moveout by Fourier transform, Geophysics, 49, 741-757, 1984.

Liner, C. L., General theory and comparative anatomy of dip moveout, Geophysics, 55, 595607, 1990.

Notfors, C. D., and Godfrey, R, J., Dip moveout in the frequency-wavenumber domain, Geophysics, 52, 1718-1721, 1987.

Zhou, B., Mason, I. M., and Greenhalgh, S. A., An accurate formulation of log-stretch dip moveout in the frequency-wavenumber domain, Geophysics, 61, 815-821, 1996.

Zhou, B., Mason, I. M., and Greenhalgh, S. A., Accurate and efficient shot-gather dip moveout processing in the log-stretch domain, Geophysical Prospecting, 43, 863-978, 1995. 